\title{
Folate deficiency and psycho-organic syndrome in elderly subjects: review of the literature
}

\author{
Flavio Cursi \\ Azienda Ospedaliera S. Camillo Forlanini, Roma, Italy
}

\begin{abstract}
Psychological syndrome is a mental disorder caused by organic brain damage accompanied by cognitive and memory deficits. According to recent estimates of incidence in the elderly population worldwide, this is likely to increase more and more over the next few years; the increase in life expectancy which in itself is a positive result, on the other hand, leads to an increase in the incidence of brain degenerative diseases that in elderly subject need to be addressed through appropriate therapeutic strategies. The lack of folates, as it is at the base of the cascade of events that lead to the development of cardio/cerebrovascular problems, anemia and depression, typical characteristics of the psycho-organic syndrome, becomes a condition that is important to correct. Several epidemiological studies show the efficacy of folates in reducing the clinical expressions typical of the syndrome, in particular the metabolic active form of folic acid, 5-methyltetrahydrofolate, is characterized by high bioavailability and rapidity of action. In clinical practice these peculiarities could be very useful both at the beginning of therapy, when it is important to have a quick and effective response, and in the maintenance, with a reduction of administration.
\end{abstract}

\section{Introduction}

The progressive aging of the population is well known. ${ }^{1}$ Recent data, related to elderly population worldwide, show that in 2000 approximately 600 million people were over 60 , while in 2025 it will be 1.2 billion, and 2 billion in $2050 .^{2}$

Correspondence: Flavio Cursi, Azienda Ospedaliera S Camillo Forlanini, Sede Legale Circ.ne Gianicolense 87, 00152 Roma, Italy.

E-mail: f.cursi63@gmail.com

Key words: Elderly; psycho-organic syndrome; 5-methyltetrahydrofolate.

Acknowledgments: medical writer assistance Dr. Valeria Battezzati.

Funding: the research is sponsored by Zambon Italia Srl.

Conflict of interest: the author declares no potential conflict of interest.

Received for publication: 1 December 2017.

Revision received: 10 April 2018.

Accepted for publication: 17 May 2018.

This work is licensed under a Creative Commons Attribution NonCommercial 4.0 License (CC BY-NC 4.0).

CCopyright F. Cursi, 2018

Licensee PAGEPress, Italy

Italian Journal of Medicine 2018; 12:116-125

doi:10.4081/itjm.2018.958
With the increase in life expectancy and the increasingly unfavorable relationship between active and non-active population, the socio-economic burden related to care and assistance for the elderly will also increase and will increase the incidence of many degenerative diseases characterized by cognitive impairment related to age. ${ }^{1}$

The presence of a picture of fragility, with progressive cognitive impairment, such as to interfere with normal daily activities, it is called a psychoorganic syndrome. ${ }^{3}$

There are several disorders of the cognitive sphere that characterize the psycho-organic syndrome in the elderly, for example: alteration of vigilance and critical judgment, difficulty with attention and concentration, loss of memory and interests, depression of mood, inability to resolve everyday problems. With regard to this current issue, there is a growing need to manage elderly subject, characterized by a profile where some inherent frailty may not be fully apparent, with a multidimensional approach.

Liotta et al. have recently published a cross-sectional study whose aim was to evaluate the prevalence of a state of frailty in the elderly population residing in the Region of Latium and to describe a method for planning ad hoc interventions. In approximately $15 \%$ of subjects subjected to multidimensional geriatric evaluation, a certain degree of frailty has been recorded, and in over $7 \%$, this grade is severe. ${ }^{4}$

Within this context, a large study is under way which aims to assess the impact of programs implemented in different European States to counter the three major consequences of age-related frailty: hospitalization, institutionalization and death. Such 
study, will give the opportunity to collect specific information on the incidence of frailty and the progression/regression of the risk of development of negative outcomes, in subjects over the age of 64 who are not institutionalized. ${ }^{5}$

Further, within the issues that are often encountered in elderly subjects, Donini et al. wanted to assess how nutritional deficiencies play a crucial role in the development of clinical and/or functional handicaps in the elderly. For this purpose, 246 subjects were enrolled (164 females of 82.3 average age, and 82 males of 76.5 average age) which received a multidimensional evaluation. Based on the outcome collected with the Mini-Nutritional Assessment questionnaire (a screening and nutritional assessment tool able to identify elderly subjects at nutritional risk or probably malnourished) $56.7 \%$ of women, and $61 \%$ of men were at risk of malnutrition and about $20 \%$ were actually malnourished; moreover, malnutrition was significantly associated with an increased risk of death. Functional, psychological and cognitive parameters were also influenced by the nutritional state of the subject. ${ }^{6}$

Vitamins are essential for the proper functioning of the organism and their deficiency can cause important dysfunctions, as in the case of folate, watersoluble vitamins of the B group. ${ }^{7,8}$

Aging is in itself a risk factor for the development of folate deficiency. As demonstrated by a large study conducted in 3511 subjects, there was a high percentage of elderly people with folate deficiency and vitamin B12, which increases according to age: from 1 in 20 cases in subjects aged 65 to 74 years to 1 in 10 among those over 75 years. ${ }^{9}$

\section{Psycho-organic syndrome due to folate deficiency in the elderly}

With regard to the underlying causes of the development of this fragile nature in the elderly, certainly their nutritional state, in which a lack of folate is detected, is an important variable on which we can intervene. Such deficiency is often caused by problems related to various factors as for example: ${ }^{8}$

- malnutrition;

- malabsorption; i) atrophic gastritis; ii) intestinal diseases (Crohn's disease, celiac disease, chronic inflammatory forms, diverticulitis/ diverticulosis); iii) gastric and/or intestinal resection;

- pharmacotherapy (antifolates, methotrexate, proton pump inhibitors, antiepileptic drugs);

- chronic liver disease and alcoholism;

- genetic polymorphisms in the enzymes involved in folate metabolism (e.g. methyl-tetrahydrofolate reductase).

Folate deficiency seems to be closely related to the development of psycho-organic problems. ${ }^{8}$ The main related symptoms are hematologic, neurological and cardiovascular. $^{7}$

Although the underlying mechanisms of psychoorganic alterations linked to folate deficiency in the elderly population are not fully understood, some hypotheses have been put forward, in particular, the defects in DNA synthesis, the metabolism of amino acids (hyperhomocysteinemia), the reduced methylation capacity (Figure 1$)^{8}$ and the reduced availability of S-adenosylmethionine (SAM).

Therefore, in the case of a psycho-organic syndrome, attention will also need to be paid to the clinical consequences of changes in blood chemistry parameters such as anemia, hyperhomocysteinemia, blood folate concentrations and vitamin B12 associated with the increase of cardiovascular, neurological and mood diseases.

\section{Anemia}

It is well known that anemia, a disease commonly found in General Medicine, is diagnosed in a higher percentage in the elderly than in non-elderly populations: approximately $5.5 \%$ in $50-64$ years old and more than $20 \%$ in over- 85 years old. Said disorder is an independent predictor of survival in elderly populations as negatively affecting their quality of life. Epidemiological studies report that in about one third of the elderly population anemia is caused by folate, iron or vitamin B12 deficiency. ${ }^{10}$

Several studies have shown that the presence of anemia in the elderly is worsening both physical and mental clinical outcomes, thus, in these subjects, it is necessary to intervene in a timely manner to handle this problem.

In particular, macrocytic anemia - or megaloblastic [mean corpuscular volume $(\mathrm{MCV}) \geq 100 \mathrm{fL}$ ] is determined by any factor that is capable of altering DNA synthesis, such as vitamin B12 and/or folate deficiency, the intake of some types of drugs that can alter DNA synthesis or antagonize folate action. The prevalence of macrocytic anemia results to be around $7.5 \%$ (data observed in a population of 3324 Dutch elderly subjects). ${ }^{11}$

\section{Hyperhomocysteinemia}

Numerous epidemiological studies show that hyperhomocysteinemia is an independent risk factor both for the development of cardiovascular and cerebrovascular diseases, as well as neurological disorders (dementia, Alzheimer's disease). ${ }^{8}$ Several dietary factors and lifestyles can affect homocysteine levels; in particular, it has been observed that a high 
folate intake is associated with a reduction in the homocysteine concentration. ${ }^{12}$

The metabolism of homocysteine occurs mainly in two ways:

i) by remethylation pathway, in which homocysteine is converted into methionine by methionine synthase (MS) in the presence of vitamin B12 (folate-dependent reaction);

ii) by transsulfuration pathway, in which homocysteine is converted into cysteine by cystathionine $\beta$-synthase in the presence of vitamin B6 (Figure 2). ${ }^{13}$

It is well known that circulating levels of homocysteine also depend on the presence of gene mutations encoding the methylenetetrahydrofolate reductase enzyme (MTHFR), a key enzyme for the transformation of 5,10-MTHF into 5-MTHF, a metabolically active compound essential for homocysteine remethylation. The impaired activity of MTHFR reduces up to $50 \%$ the actual activity of the enzyme and results in an increase of approximately $25 \%$ of homocysteine levels, due to lack of reconversion into methionine. ${ }^{14,15}$

Polymorphism of the MTHFR gene $(677 \mathrm{C} \rightarrow \mathrm{T})$ causes an amino acid variation: an alanine is replaced by a valine and, in the case of a mutation in homozygous recessive form, there is a reduction in the stability of the enzyme and its activity.

This type of mutation is widespread worldwide: in Europe, over $12 \%$ of the population is homozygous TT, $43 \%$ is heterozygous CT and $45 \%$ is wild-type CC. In Italy, the incidence of TT homozygosity is even higher than the European average: $18 \%$ in the North, $20 \%$ in Central Italy, and $26 \%$ in the South. ${ }^{15,16}$

For indicative purposes, in adults the normal levels of homocysteine are considered between 6 and 10 $\mu \mathrm{mol} / \mathrm{L} .{ }^{17}$

A consensus paper published in 2003, defined hyperhomocysteinemia, classifying it according to plasma concentrations, as follows: i) moderate 15-30 $\mu \mathrm{mol} / \mathrm{L}$; ii) intermediate $30-100 \mu \mathrm{mol} / \mathrm{L}$; iii) severe $>100 \mu \mathrm{mol} / \mathrm{L}^{17}$

\section{Hyperhomocysteinemia and cardiovascular disorders}

Numerous epidemiological studies have reported a significant increase in the risk of coronary heart disease, myocardial infarction, stroke, venous thromboembolism and peripheral vascular disease in patients with elevated homocysteine levels. ${ }^{13}$

A first meta-analysis, published in 1995 that

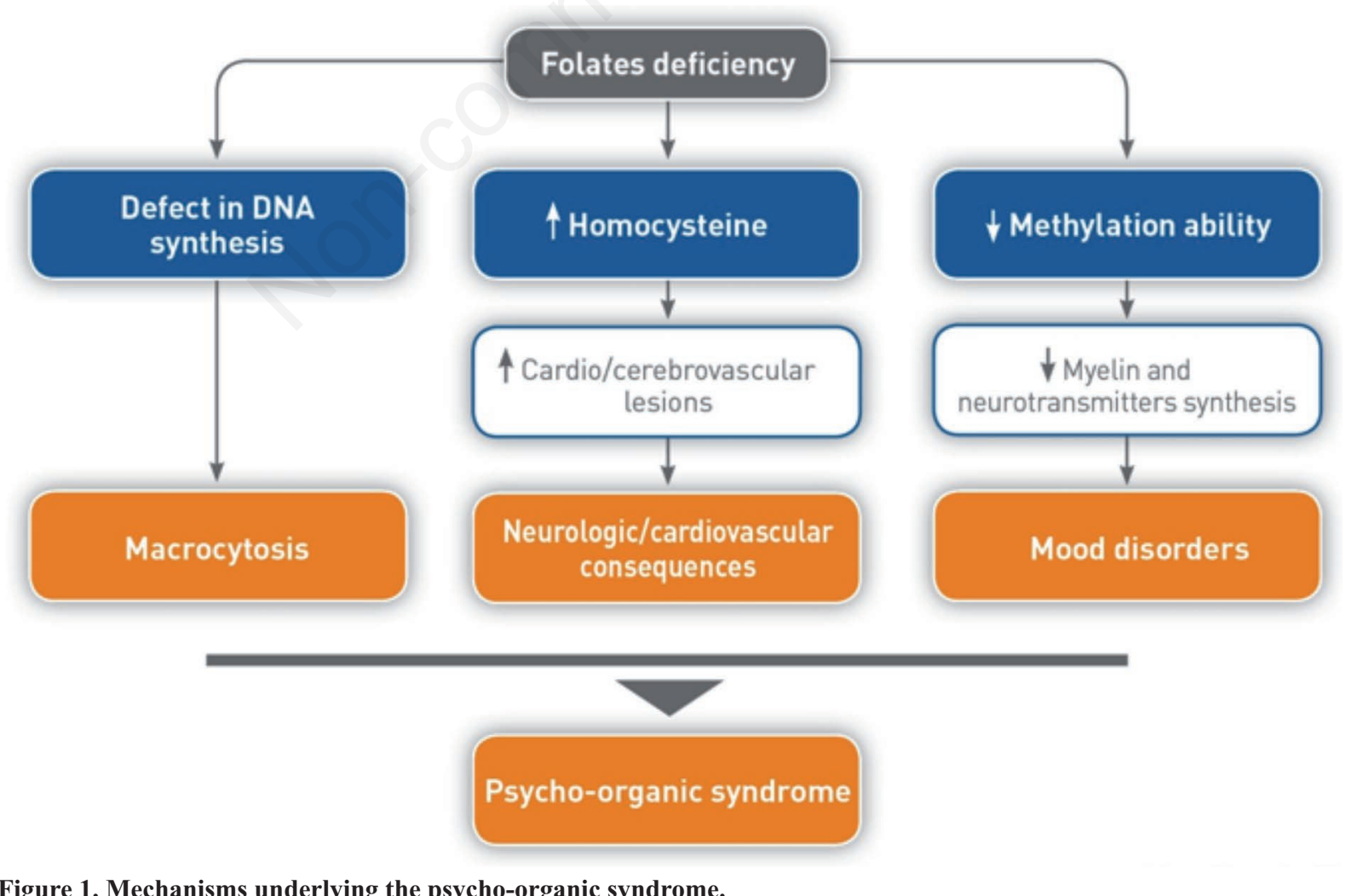

Figure 1. Mechanisms underlying the psycho-organic syndrome. 
included 27 retrospective and prospective studies, reports an increased risk of coronary heart disease for each increment of $5 \mathrm{mmol} / \mathrm{L}$ of total homocysteine [men: odds ratio $(\mathrm{OR})=1.6 ; \%$ 1.4-1.7; women: $\mathrm{OR}=1.8 ; 95 \%$ confidence interval (CI) 1.3 1.9]. From this result, the authors extrapolate that $10 \%$ of the risk of developing coronary heart disease in the global population is attributable to hyperhomocysteinemia, and that up to 50,000 deaths/year could be avoided with a reduction in the level of homocysteine. ${ }^{18}$

These conclusions were supported by the results of a meta-analysis of the Homocysteine Studies Collaboration where a reduction of the risk of $11 \%$ ischemic heart disease and a stroke of $19 \%$ is observed with a $3 \mathrm{mmol} / \mathrm{L}$ reduction in the homocysteine concentration. ${ }^{19}$

Stanhewicz et al. have recently published a review highlighting the role of 5-MTHF, an active metabolite of folic acid, in the cascade of events leading to increased production and bioavailability of nitric oxide (NO) at the endothelial level. ${ }^{20}$

Specifically, said 5-MTHF action is due to its ability to act at an isoform endothelial level of the nitric oxide synthase enzyme (eNOS). Said enzyme performs its regulatory function on vascular tone only if it is properly assembled in its subunits (eNOS in a coupling state), and in the presence of appropriate concentrations of the tetrahydrobiopterin cofactor (BH4). In contrast, in oxidative stress conditions, the enzyme structure is altered (eNOS is in a decoupling state) and becomes a superoxide anion that removes $\mathrm{NO}$, and produces highly cytotoxic peroxynitrite. ${ }^{21}$

Homocysteine affects vascular function by indirect action on vascular tone, which induces greater vasoconstriction. Under normal conditions, homocysteine reacts with NO forming a nitrosothiol with vasodilating activity that prolongs the fleeting effect of NO. When homocysteine is in excess, is no longer normally neutralized by NO, undergoes the homocysteine self-oxidation process, which simultaneously produces oxygen highly reactive ions and free radicals reactive oxygen species (ROS). Oxidative stress triggers eNOS inversion from NO generator to superoxide generator, accentuating oxidative stress; the endothelial bioavailability of NO as vasodilation mediator, therefore decreases. The consequence is that the subject experiences continuous vasospasms, a phenomenon well-known in patients with kidney failure. ${ }^{21}$

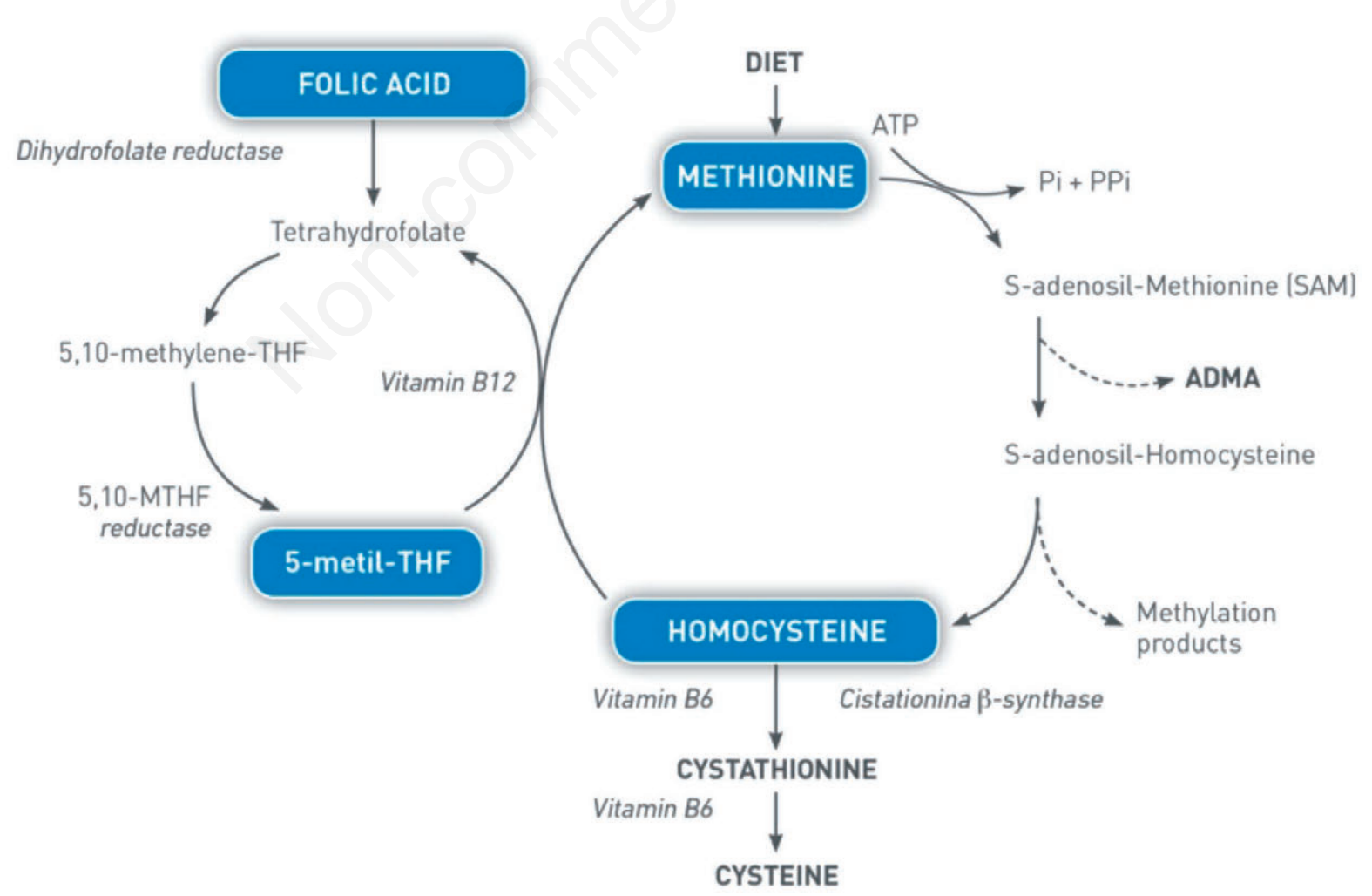

Figure 2. Cycle of homocysteine metabolism. ADMA, asymmetric dimethyl-arginine; THF, tetra-hydro-folate; MTHF, methyl-hydrofolate reductase. Adapted from Maron and Loscalzo et al., $2009^{13}$ with permission. 
The formation of atherosclerotic plaque is triggered by a direct damage mechanism: the endothelium increases the proliferation of smooth muscle cells induced by the oxidative state with high circulating concentrations of homocysteine, parallel to an interference with the normal platelet function. ${ }^{17}$ This is because excess homocysteine forms the homocysteine-thiolactone complex that reacts with low-density lipoproteins (LDL) forming an insoluble LDL-thiolactone complex that is phagocytized by macrophages. The latter, being unable to shed it, become foam cells forming the core of the atheroma. Excess homocysteine can also act as an oxygen free radical, causing:

i) endothelial dysfunction and then necrosis in endothelial cells with their detachment from vessel walls;

ii) proliferation of smooth muscle cells with subsequent fibrosis and fibrocalcification of the vessel area;

iii) oxidation of membrane lipids with loss of functionality of these structures;

iv) oxidation of LDLs, with onset of diffuse atherogenesis.

Further, NO actions include the inhibition of migration and proliferation of smooth muscle cells, so that the direct action of homocysteine may also be associated with the indirect action, also attributable to NO depletion. ${ }^{21}$

\section{Hyperhomocysteinemia and neurological disorders}

Seshardi et al. first published on the New England Journal of Medicine a prospective epidemiological evaluation with the aim of verifying the possible correlation between Alzheimer's dementia/disease and homocysteine levels. Data from the Framingham study of 1092 non-demented subjects (667 women and 425 men, of 76 average age) monitored for over eight years was considered. During the follow-up period, 111 subjects showed a clinical presentation of dementia, and among these, 83 of them were diagnosed with Alzheimer's disease. With reference to the homocysteinemia and dementia ratio, the authors noted that the risk of developing Alzheimer's disease doubled in patients with homocysteine levels $>14 \mu \mathrm{mol} / \mathrm{L} .^{22}$

High levels of homocysteine may therefore be considered as an independent risk factor and strong predictors of development of dementia and/or Alzheimer's disease in the elderly. ${ }^{22}$

Similar data were also observed in other populations and confirmed in the Conselice Study of Brain Aging Italian population study, in which 816 elderly subjects were enrolled (with an average age of 74) in whom, during a 4-year follow-up period, dementia was diagnosed in 112 subjects, of whom 70 with Alzheimer's disease. In subjects with plasma homocysteine levels $>15 \mathrm{mmol} / \mathrm{L}$ there was a risk of dementia development twice as high than in the normal homocysteine level population. ${ }^{23}$

Regardless of homocysteine, low levels of folate such as $\leq 11.8 \mathrm{nmol} / \mathrm{L}$, were also found to be a significant independent risk factor for dementia/ Alzheimer's disease development. ${ }^{23}$

Several hypotheses have been established to explain the correlation between the development of dementia-type pathologies and the levels of homocysteine circulating; one of which involves the GABA receptor of central nervous system cells, which is antagonized by the presence of high levels of homocysteine which also promotes the production of ROS causing the apoptosis or cell death typical of such pathologies. ${ }^{24}$

\section{Hyperhomocysteinemia and mood disorders}

The high concentration of homocysteine is also associated with the onset of depression..$^{25}$

A meta-analysis of nine observational studies, conducted on 3752 subjects aged $\geq 70$, showed a direct correlation between homocysteine levels and the onset of depression: subjects with homocysteine $\geq 12.5$ $\mathrm{mmol} / \mathrm{L}$ exhibited a $70 \%$ higher risk. ${ }^{26}$

Opolska et al. also found that more than $50 \%$ of depressed patients have high levels of homocysteine, ${ }^{27}$ but also reduced levels of folate are associated with an increased risk of depression. ${ }^{8}$

It is also interesting to note that in patients with depression, homocysteine levels are significantly associated with the different MTHFR genotype; in subjects with CT or TT genotype, the prevalence of hyperhomocysteinemia and depression was higher. ${ }^{28}$

The pathophysiological mechanism underlying the development of mood disorders, in the case of hyperhomocysteinemia and folate deficiency, appears to be linked to the fact that homocysteine is remethylated to methionine in a lower amount and this results in a decrease in SAM levels, a key ingredient in the synthesis of essential neuromediators for the person's psychological equilibrium such as dopamine, norepinephrine, epinephrine and serotonin. Additionally, homocysteine contributes to the production of neurotoxic compounds such as homocysteic acid and cysteinesulfinic acid, which acts as an antagonist of N-methyl-D-aspartate receptors and has neurotoxic effects on dopaminergic neurons. ${ }^{29}$

\section{Management of psycho-organic syndrome caused by folate deficiency in the elderly}

In frail subjects such as the elderly, it is shown that folate deficiency may be related to the development of cardio/cerebrovascular problems, anemia and 
depression; this is because folates are at the base of the cascade of events that determine these conditions. ${ }^{7}$

It is therefore evident the importance of adequate intake of folates, given that man does not have the ability to synthesize them. ${ }^{7}$

These substances can be taken with food (e.g., spinach, broccoli, asparagus, lettuce, beans, peas, kiwis, strawberries, oranges, almonds, walnuts, liver, eggs), or by pharmacologic supplementation. ${ }^{30}$ Among the different formulations, the availability of 5-MTHF active folate supplements can be advantageous, as ready to use (5-MTHF is used by the body as such without undergoing further biotransformation processes), it is readily absorbed by digestive tract and is distributed to all tissues including the central nervous system (CNS) and with a greater rapidity of action. ${ }^{31}$

\section{Macrocytic anemia and folate supplementation}

Documented anemia diagnosis (hemoglobin $<12$ $\mathrm{g} / \mathrm{dL}$ in men and $<13 \mathrm{~g} / \mathrm{dL}$ in women), MCV $>100 \mathrm{fL}$ and high levels of homocysteine are significant indicators for the use of folates in the elderly; this recommendation of use has also been suggested by Bross et al..$^{32}$

\section{Hyperhomocysteinemia, cardiovascular disorders and folate supplementation}

The Homocysteine Lowering Trialists' Collaboration has recently published a meta-analysis with the aim of verifying the minimum dose of folate to be administered to achieve maximum reduction of plasma homocysteine levels. For this purpose, 25 randomized trials were screened, with the involvement of 2596 subjects of an approximate average age of 55 (maximum age 92, 46\% men, 30\% with a cardiovascular event history); the average duration of treatment was $8 \pm 6$ weeks.

A dose-dependent reduction of up to $25 \%$ homocysteine levels was observed with a daily dose of $5 \mathrm{mg}$ folic acid (Figure $3^{19}$ ). ${ }^{33}$

The administration of vitamin B12, $0.4 \mathrm{mg} /$ day, resulted in a $7 \%$ reduction (CI at $95 \%$ : $4 \%-9 \%$ ) of plasma homocysteine levels while no change was observed with the use of vitamin B6.

In addition, a decrease in hyperhomocysteinemia was observed proportionally based on baseline parameters. ${ }^{33}$

Ambrosino et al. recently published a prospective study aimed at assessing the hypothesis that a cyclical supplement (one month of therapy followed by two months of interruption) with 5-MTHF could reduce total plasma levels of fasting homocysteine in recently diagnosed, treatment naïve patients to folate treatment or 5-MTHF, with mild hyperhomocysteinemia, or moderate hyperhomocysteinemia.

5 -MTHF, $15 \mathrm{mg}$ /day was administered to 228 patients for a period of two years according to the following schedule: one month of therapy followed by two months of interruption.

After one 5-MTHF treatment cycle, both a reduction of $54.4 \%$ of total homocysteine levels (from $31.6 \mu \mathrm{mol} / \mathrm{L}$ at baseline to $14.4 \mu \mathrm{mol} / \mathrm{L}, \mathrm{P}<0.001 v s$ baseline), and a parameter normalization were observed, in over $50 \%$ of patients $(51.3 \%)$. The best outcomes were recorded after eight cycles of supplementation (two years); in fact, both a reduction of $61.5 \%$ of total homocysteine levels (from 31.6

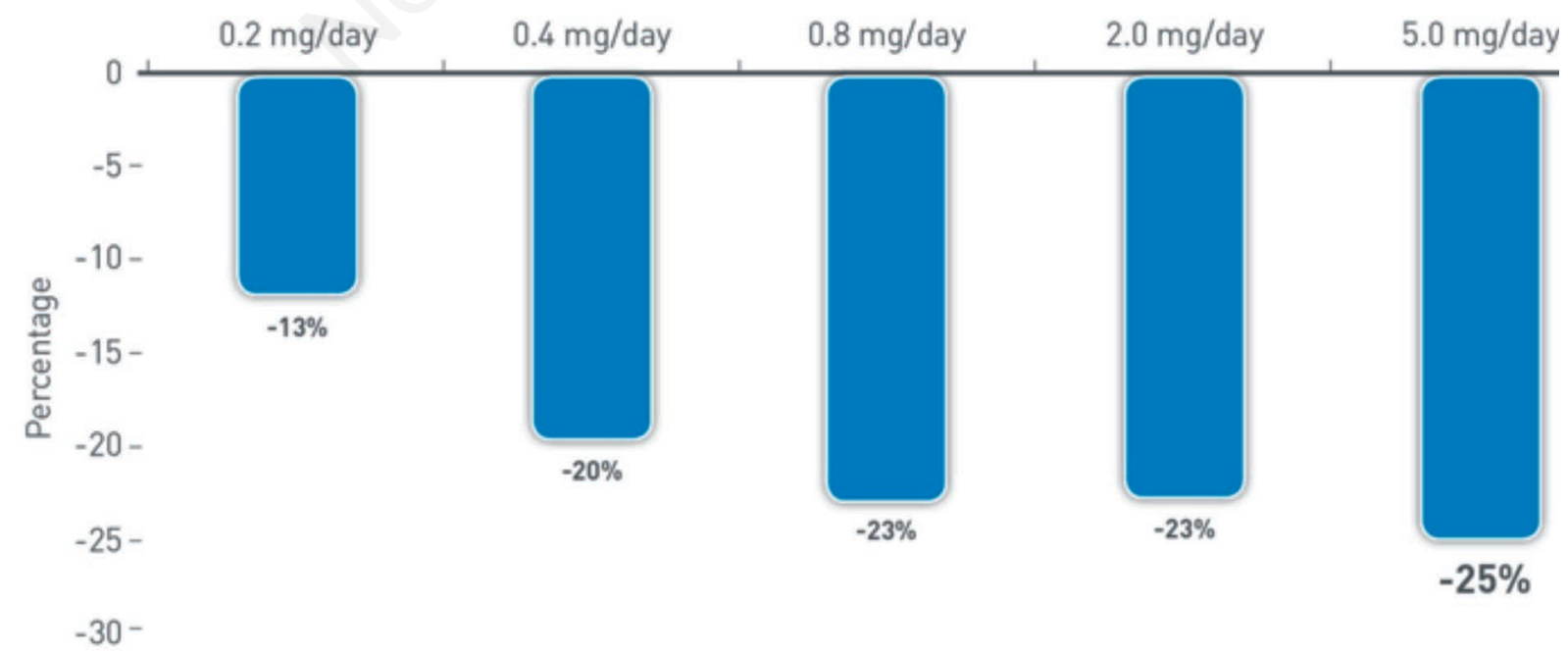

Figure 3. Reduction in plasma homocysteine levels based on different folate doses. Adapted from Homocysteine Studies Collaboration, 2002. ${ }^{19}$ with permission. 
$\mu \mathrm{mol} / \mathrm{L}$ at baseline to $12.18 \mu \mathrm{mol} / \mathrm{L} ; \mathrm{P}<0.001 v s$ baseline), and a normalization of the parameter in nearly $90 \%$ of patients $(86.8 \%)$ were recorded.

The average number of treatment cycles needed to achieve normalization of total homocysteine levels was 1.78. Said parameter varied according to patient conditions, namely presence or absence of an MTHFR mutation, with or without metabolic syndrome. ${ }^{34}$

The outcome of this single-panel prospective study shows that one month of 5-MTHF therapy followed by two months of interruption (to be repeated cyclically) is able to significantly reduce total homocysteine levels in patients with mild to moderate hyperhomocysteinemia. In over $50 \%$ of patients, total homocysteine levels became normalized within the first treatment cycle and in $86.8 \%$ of patients during the following two years of treatment. In addition, the presence of metabolic syndrome and MTHFR mutation in homozygosity are considered negative predictors of response to 5-MTHF supplementation; thus, there is a need for more supplementation cycles to reach the total target homocysteine levels. ${ }^{34}$

The impact of folate administration in patients with cardiovascular disease does not end with the reduction of homocysteine, but involves wider effects that lead to an improvement in endothelial function and oxidative stress. The mechanisms responsible for these effects include direct antioxidant properties of 5-MTHF and an interaction with the eNOS enzyme. ${ }^{21}$

\section{Hyperhomocysteinemia, neurological disorders and folate supplementation}

It is well known, that hyperhomocysteinemia can affect brain function both by its action at vascular level and its neurotoxicity; further, low levels of B-complex vitamins reduce the production of S-adenosylmethionine, an important mediator of brain methylation reactions involved in synaptic transmission. In addition, tetrahydrofolate is involved in DNA repair and DNA replication, which is a fundamental process for adult hippocampal neurogenesis. ${ }^{35}$

In a large panel of elderly French subjects (2104, non-institutionalized) $>65$ years of age, monitored over a decade, high levels of folate intake have been inversely associated with the risk of developing dementia. Compared to subjects with folate supplementation in the lowest quintile, those in the highest quintile had an approximately $50 \%$ lower risk of dementia. ${ }^{35}$

These results are consistent with at least three previous longitudinal studies performed in the United States which, even in these cases, showed that with higher folate intake there was a lower risk of onset of dementia or Alzheimer's disease. ${ }^{36}$ In Italy, a pilot study highlighted the benefit of active 5-MTHF folate supplementation, on memory and attention on elderly subjects selected with low folate baseline values. ${ }^{37}$

Other studies in the Netherlands, which included subjects of an age $\geq 50$ with hyperhomocysteinemia, showed a significant improvement in memory and processing speed with folic acid supplementation for three years. ${ }^{38}$

\section{Mood disorders and folate supplementation}

A folate deficiency results in the limited conversion of homocysteine into methionine, the essential precursor amino acid of SAM. SAM plays a key role in the methylation of neurotransmitters involved in the development of depression, such as choline, serotonin, dopamine, and noradrenaline. ${ }^{25}$

Based on this premise, SAM concentrations and metabolites of monoamine neurotransmitters in the cerebrospinal fluid of severely depressed patients were evaluated. As expected, elevated levels of these compounds as well as homocysteine were detected. ${ }^{39}$

Some randomized clinical trials have evaluated the effect of folic acid supplementation in the prevention and progression of neuropsychiatric disorders in the elderly. Durga et al. conducted a study in 818 adult subjects aged between 50 and 70 and with plasma homocysteine concentrations $\geq 13 \mathrm{mmol} / \mathrm{L}$. Three-year supplementation with folic acid $800 \mu \mathrm{g} /$ day resulted in an increase in plasma folate levels of $576 \%$ (CI of $95 \%$ : $539 \%-614 \%$ ) and a $26 \%$ total plasma homocysteine reduction (range: 24\%-28\%), compared with placebo. In addition, an improvement of memory, information processing speed, and sensorimotor speed significantly higher than in the placebo, was observed. ${ }^{38}$

Further, in patients over 55 with depression and folate deficiency, 5-MTHF supplementation has been shown to improve mood/depression symptoms and short-term memory. ${ }^{8}$

As long ago as 1993 Passeri et al., in a doubleblind randomized, eight-week study, in which 96 patients with depression were treated with 5-MTHF, $50 \mathrm{mg} /$ day $(\mathrm{n}=47)$, or trazodone, $100 \mathrm{mg} /$ day $(\mathrm{n}=49)$, demonstrated a significant clinical and depressive symptoms improvement, compared to baseline for both treatments.

After four weeks of therapy, the Hamilton Rating Scale for Depression score was significantly reduced with 5-MTHF supplementation to a slightly higher degree than with trazodone treatment. A further significant improvement was observed at the end of the study (eight weeks). ${ }^{40}$

Other authors report a noteworthy improvement in symptoms when antidepressant or cholinesterase 
inhibitor treatment is associated with folate supplementation in patients with depression. ${ }^{8}$

Lastly, Almeida et al. conducted a (randomized, double-blind, controlled) trial in depressed patients over the age of 55, with the aim of verifying whether vitamin B6 (25 mg), B12 (0.5 mg) and folic acid (2 mg) for over 52 weeks was able to improve response to treatment with the antidepressant citalopram (20-40 g).

A total of 153 patients were randomized (citalopram + placebo: $n=76$; citalopram + vitamins: $\mathrm{n}=77$ ). Remission of symptoms was observed at week 12 in $78.1 \%$ and $79.4 \%$ of participants treated with citalopram + placebo and with citalopram + vitamins $(\mathrm{P}=0.840)$, respectively. Similar results were obtained after 26 and 52 weeks. ${ }^{41}$

The risk of further recurrence, in patients in remission at week 12, was lower in the group treated with citalopram + vitamins compared with citalopram + placebo. $^{41}$

These beneficial effects were apparent in patients with high baseline levels of homocysteine. ${ }^{41}$

\section{Conclusions}

Over the next few years, our society will have to deal more and more with the issue of increasing longevity and consequently the issues related to the elderly, such as the psycho-organic syndrome, characterized by organic brain damage accompanied by a decrease in intellectual abilities, impaired alertness and critical judgment, difficulty in attention, deficit of memory. The nutritional deficiencies, and in particular the folate deficiency, have an important role in the cascade of events involved in the onset of the typical characteristics of the psycho-organic syndrome; this is why it is important to intervene promptly.

There are several observational or randomized epidemiological studies that highlight the efficacy of folate and 5-MTHF, a metabolically active form of folic acid, in reducing the typical clinical manifestations of psycho-organic syndrome.

Unfortunately, many studies in the cardiovascular field have used the benchmark of folic acid blood concentration in an uncritical manner. In fact, the reduction of homocysteine, in the case of hyperhomocysteinemia, is not directly proportional to the increase in blood concentration of folic acid. It can be seen from the above, how a number of correlations in recent clinical trials have led to erroneous conclusions (as documented by Cochrane's review) that have reshaped the use of non-activated folic acid in the cardiovascular field. Currently, further studies with 5-MTHF, particularly in elderly subjects, are still ongoing, which tend to show how only active forms of folic acid are effective. ${ }^{42}$

By analogy with other commonly used therapies such as vitamin D supplementation, where the choice of the single product is customized to the characteristics of the subject to whom it is administered (renal failure, liver disease, nutritional deficiency), so folate supplementation should follow rational assumptions. For this reason, in clinical practice, in the case of the elderly person, it may be useful to always start the therapy with 5-MTHF, which, at present, represents the only ready-to-use therapeutic opportunity. ${ }^{43}$

The advantage of using 5-MTHF compared to folic acid consists of its high bioavailability (seven times higher regardless of the MTHFR genotype), and rapid action, with the consequent possibility of reducing administration for maintenance in the medium-term. ${ }^{31}$

Compared to folic acid, the benefits of the use of 5-MTHFR are manifold: reduction of administration, active metabolite, high bioavailability ( 7 times higher independently of the MTHF genotype), distribution to the CNS.

Therefore, in our daily clinical practice, in the patient with cardiovascular diseases, cognitive and nutritional deficits, inflammatory bowel disease, polypharmacy (in particular in the presence of antiepileptic drugs, neuroleptics, mood stabilizers) diabetes mellitus, chronic renal failure, inflammatory diseases (also of rheumatologic competence with constantly altered inflammatory indexes), anemia of any origin, administration of 5-MTHF should be taken into consideration.

In conclusion, also in light of the most recent scientific evidence (such as the dopaminergic hypothesis of the D'Amelio group) the administration of 5-MTHF must become a first-choice therapeutic option to prevent or reduce the incidence of psychoorganic syndromes. ${ }^{44}$

\section{References}

1. Galluzzo L, Gandin C, Ghirini S, Scafato E. L'invecchiamento della popolazione: opportunità o sfida? Available from: http://www.epicentro.iss.it/ben/2012/ aprile/2.asp Accessed: 15/1/2018.

2. World Health Organization (WHO). The Global strategy and action plan on ageing and health. Available from: http://www.who.int/ageing/global-strategy/en/ Accessed: 15/1/2018.

3. Zangara A. Medicina preventiva e riabilitativa. Padova: Piccin Nuova Libraria Spa; 1996.

4. Liotta G, O'Caoimh R, Gilardi F, et al. Assessment of frailty in community-dwelling older adults residents in the Lazio region (Italy): A model to plan regional community-based services. Arch Gerontol Geriatr 2017;68:1-7.

5. Liotta G, Orfila F, Vollenbroek-Hutten M, et al. The European innovation partnership on active and healthy ageing synergies: protocol for a prospective 
observational study to measure the impact of a community-based program on prevention and mitigation of frailty (ICP - PMF) in community-dwelling older adults. Transl Med UniSa 2016;15:53-66.

6. Donini LM, Poggiogalle E, Molfino A, et al. Mininutritional assessment, malnutrition universal screening tool, and nutrition risk screening tool for the nutritional evaluation of older nursing home residents. J Am Med Dir Assoc 2016;17:959.

7. Lökk J. News and views on folate and elderly persons. J Gerontol A Biol Sci Med Sci 2003;58:354-61.

8. Araújo JR, Martel F, Borges N, et al. Folates and aging: Role in mild cognitive impairment, dementia and depression. Ageing Res Rev 2015;22:9-19.

9. Clarke R, Grimley Evans J, Schneede J, et al. Vitamin B12 and folate deficiencyin later life. Age and Ageing 2004;33:34-41.

10. Guralnik JM, Eisenstaedt RS, Ferrucci L, et al. Prevalence of anemia in persons 65 years and older in the United States: evidence for a high rate of unexplained anemia. Blood 2004;104:2263-8.

11. Stouten K, Riedl JA, Droogendijk J, et al. Prevalence of potential underlying aetiology of macrocytic anaemia in Dutch general practice. BMC Fam Pract 2016;17:113.

12. Rasmussen LB, Ovesen L, Bülow I. Folate intake, lifestyle factors, and homocysteine concentrations in younger and older women. Am J Clin Nutr 2000;72: 1156-63.

13. Maron BA, Loscalzo J. The treatment of hyperhomocysteinemia. Annu Rev Med 2009;60:39-54.

14. Pietrzik K, Bailey L, Shane B. Folic acid and L-5methyltetrahydrofolate: comparison of clinical pharmacokinetics and pharmacodynamics. Clin Pharmacokinet 2010;49:535-48.

15. Scaglione F, Panzavolta G. Folate, folic acid and 5methyltetrahydrofolate are not the same thing. Xenobiotica 2014;44:480-8.

16. Wilcken B, Bamforth F, Li Z et al. Geographical and ethnic variation of the $677 \mathrm{C}>\mathrm{T}$ allele of 5,10 methylenetetrahydrofolate reductase (MTHFR): findings from over 7000 newborns from 16 areas worldwide. J Med Genet 2003;40:619-25.

17. Pezzati P, Balboni F, Graziani MS. Iperomocisteinemia e rischio cardiovascolare: stato dell'arte. Biochim Clin 2010;34:3:173-86.

18. Boushey CJ, Beresford SA, Omenn GS, et al. A quantitative assessment of plasma homocysteine as a risk factor for vascular disease. Probable benefits of increasing folic acid intakes. JAMA 1995;274:1049-57.

19. Homocysteine Studies Collaboration. Homocysteine and risk of ischemic heart disease and stroke: a metaanalysis. JAMA 2002;288:2015-22.

20. Stanhewicz AE, Kenney WL. Role of folic acid in nitric oxide bioavailability and vascular endothelial function. Nutr Rev 2017;75:61-70.

21. Coppola A, De Simone C, Coppola D, et al. Omocisteina, folati e patologia tromboembolica. Riv Ital Ostetr Ginecol 2004;1:14-9.

22. Seshardi S, Beiser A, Selhub J, et al. Plasma homocysteine as a risk factor for dementia and Alzheimer's disease. N Engl J Med 2002;346:476-83.

23. Ravaglia G, Forti P, Maioli F, et al. Homocysteine and folate as risk factors for dementia and Alzheimer disease. Am J Clin Nutr 2005;82:636-43.

24. Ansari R, Mahta A, Mallack E, Jun Luo J. Hyperchomocysteinemia and neurologic disorders: a review. J Clin Neurol 2014;10:281-8.

25. De Koning EJ, van der Zwaluw NL, van Wijngaarden JP, et al. Effects of two-year vitamin B12 and folic acid supplementation on depressive symptoms and quality of life in older adults with elevated homocysteine concentrations: additional results from the B-PROOF Study, an RCT. Nutrients 2016;8:pii:E748.

26. Almeida OP, McCaul K, Hankey GJ, et al. Homocysteine and depression in later life. Arch Gen Psychiatry 2008;65:1286-94.

27. Opolska A, Karakula H, Kowal A, et al. Comparison of serum homocysteine levels between patients with depression and control group members - preliminary report. Pol J Environ Stud 2006;15:523-7.

28. Kang HJ, Stewart R, Bae KY, et al. Predictive value of homocysteine for depression after acute coronary syndrome. Oncotarget 2016;7:69032-40.

29. Bhatia P, Singh N. Homocysteine excess: delineating the possible mechanism of neurotoxicity and depression. Fundam Clin Pharmacol 2015;29:522-8.

30. Ruggeri S. Biodisponibilità e livelli di assunzione dei folati e dell'acido folico. In: EpiCentro. Acido folico e folati. Available from: http://www.epicentro.iss.it/problemi /folico/Biodisponibilita.asp Accessed: 15/1/2018.

31. Willems FF, Boers GH, Blom HJ et al. Pharmacokinetic study on the utilisation of 5-methyltetrahydrofolate and folic acid in patients with coronary artery disease. $\mathrm{Br} \mathrm{J}$ Pharmacol 2004;141:825-30.

32. Bross MH, Soch K, Smith-Knuppel T. Anemia in older persons. Am Fam Physician 2010;82:480-7.

33. Homocysteine Lowering Trialists' Collaboration. Dosedependent effects of folic acid on blood concentrations of homocysteine: a meta-analysis of the randomized trials. Am J Clin Nutr 2005;82:806-12.

34. Ambrosino P, Lupoli R, Di Minno A, et al. Cyclic supplementation of 5-MTHF is effective for the correction of hyperhomocysteinemia. Nutr Res 2015;35: 489-95.

35. Lefèvre-Arbogast S, Féart C, Dartigues JF, et al. Dietary $\mathrm{B}$ vitamins and a 10-year risk of dementia in older persons. Nutrients 2016;8:61-72.

36. Agnew-Blais JC, Wassertheil-Smoller S, Kang JH, et al. Folate, vitamin B-6, and vitamin B-12 intake and mild cognitive impairment and probable dementia in the Women's Health Initiative Memory Study. J Acad Nutr Diet 2015;115:231-41.

37. Fioravanti M, Ferrario E, Massaia M, et al. Low folate levels in the cognitive decline of elderly patients and the efficacy of folate as a treatment for improving memory deficits. Arch Gerontol Geriatr 1998;26:1-13.

38. Durga J, van Boxtel MP, Schouten EG, et al. Effect of 3 -year folic acid supplementation on cognitive function in older adults in the FACIT trial: a randomised, double blind, controlled trial. Lancet 2007;369:208-16.

39. Bottiglieri T, Laundy M, Crellin R, et al. Homocysteine, folate, methylation, and monoamine metabolism in depression. J Neurol Neurosurg Psychiatry 2000;69: 228-32.

40. Passeri M, Cuccinotta D, Abate G et al. Oral 5'methyltetrahydrofolic acid in senile organic mental 
disorders with depression: results of a double-blind multicenter study. Aging (Milano) 1993;5:63-71.

41. Almeida O, Ford AH, Hirani V, et al. B vitamins to enhance treatment response to antidepressants in middle-aged and older adults: results from the BVITAGE randomised, double-blind, placebo-controlled trial. Br J Psychiatry 2014;205:450-7.

42. Malouf R, Grimley Evans J, Areosa Sastre A. Folic acid with or without vitamin B12 for cognition and dementia. Cochrane Database Syst Rev 2003; CD004514.

43. Fava M, Mischoulon D. Folate in depression: efficacy, safety, differences in formulations, and clinical issues. J Clin Psychiatry 2009;70:12-7.

44. Nobili A, Latagliata EC, Viscomi MT, et al. Dopamine neuronal loss contributes to memory and reward dysfunction in a model of Alzheimer's disease. Nature Commun 2017;8:14727. 